\title{
ON THE "MANDELBROT SET" FOR A PAIR OF LINEAR MAPS AND COMPLEX BERNOULLI CONVOLUTIONS
}

\author{
BORIS SOLOMYAK AND HUI XU
}

\begin{abstract}
We consider the family of self-similar sets $A_{\lambda}$, attractors of the iterated function system $\{\mathbb{C} ; \lambda z-1, \lambda z+1\}$, depending on a parameter $\lambda$ in the open unit disk. First we study the set $\mathcal{M}$ of those $\lambda$ for which $A_{\lambda}$ is connected. We show that a non-trivial portion of $\mathcal{M}$ near the imaginary axis is the closure of its interior (it is conjectured that $\mathcal{M} \backslash$ $\mathbb{R}$ is contained in the closure of its interior). Next we turn to the sets $A_{\lambda}$ themselves and natural measures $\nu_{\lambda}$ supported on them. These measures are the complex analogs of much-studied infinite Bernoulli convolutions. Extending the results of Erdős and Garsia, we demonstrate how certain classes of complex algebraic integers give rise to singular and absolutely continuous measures $\nu_{\lambda}$. Next we investigate the Hausdorff dimension and measure of $A_{\lambda}$, for Lebesgue-a.e. $\lambda \in \mathcal{M}$, and obtain partial results on the absolute continuity of $\nu_{\lambda}$, for a.e. $\lambda$ with $|\lambda|>1 / \sqrt{2}$.
\end{abstract}

\section{INTRODUCTION}

Consider a family of iterated function systems (IFS) in the complex plane $\{\mathbb{C} ; \lambda z-1, \lambda z+1\}$ depending on a parameter $\lambda \in \mathbb{D}:=\{z \in \mathbb{C}:|z|<1\}$. Let $A_{\lambda}$ denote the attractor of the IFS, that is, $A_{\lambda}$ is the unique non-empty compact set such that

$$
A_{\lambda}=\left(-1+\lambda A_{\lambda}\right) \cup\left(1+\lambda A_{\lambda}\right)
$$

These are among the most basic self-similar sets in the plane, and there is considerable interest in understanding their topological and "fractal" properties.

There is a fundamental dichotomy for attractors of IFS with two maps: they are either connected or totally disconnected, depending on whether the sets in the right-hand side of (1.1) have non-empty intersection. Consider

The authors were supported in part by NSF grant \#DMS 0099814

2000 Mathematics Subject Classification. Primary 28A80; Secondary 28A78, 37F45, 11R06, 26C10. 
the set

$$
\mathcal{M}:=\left\{\lambda \in \mathbb{D}: A_{\lambda} \text { is connected }\right\} .
$$

It was first introduced by Barnsley and Harrington [何, who called it the "Mandelbrot set for the pair of linear maps," by analogy with the classical Mandelbrot set in complex dynamics. In fact, there are some parallels with the quadratic family $z^{2}+c$, the attractors $A_{\lambda}$ being analogs of Julia sets, see [1]. The set $\mathcal{M}$ was studied in [5, 15, 14, 29, 田, but some of the basic questions on the geometry and topology of $\mathcal{M}$ remain open. See Figure 1, made by $\mathrm{C}$. Bandt, which shows the part of $\mathcal{M}$ in $\{z: \operatorname{Re}(z)>0, \operatorname{Im}(z)>$ $0,|z| \leq 1 / \sqrt{2}\}$. Computer pictures suggest many interesting features, but it is challenging to prove them rigorously. Our first result is a step toward proving that $\mathcal{M} \backslash \mathbb{R}$ is contained in the closure of its interior, as conjectured by Bandt [1].

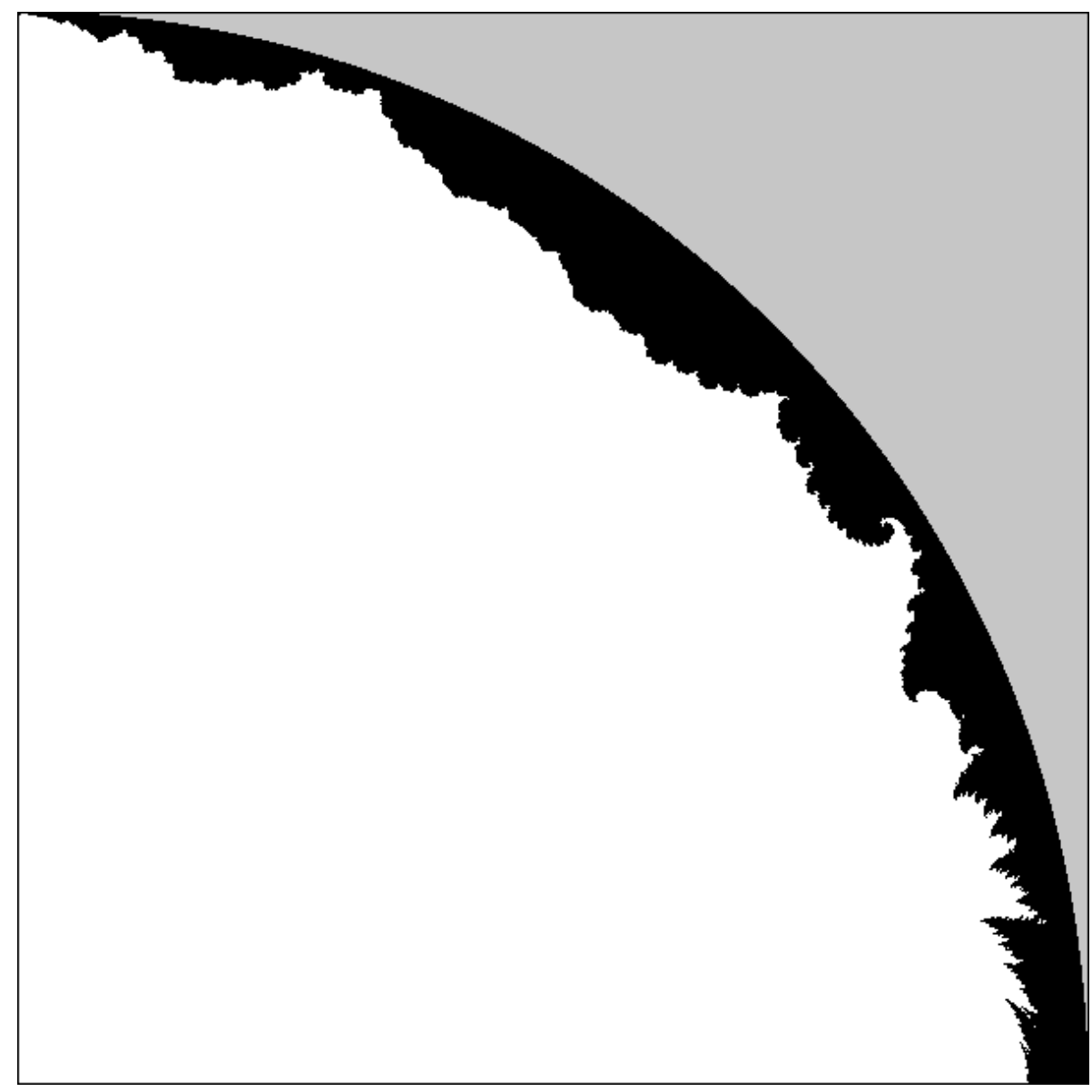

Figure 1. The "Mandelbrot set" $\mathcal{M}$ 
Next we turn to the attractors $A_{\lambda}$. The case $\lambda \notin \mathcal{M}$ is completely understood, since then $A_{\lambda}$ is totally disconnected and has Hausdorff dimension equal to the similarity dimension $s(\lambda):=\frac{\log 2}{-\log |\lambda|}$, with the corresponding Hausdorff measure positive and finite. Thus, we focus our attention on the attractors $A_{\lambda}$ for $\lambda \in \mathcal{M}$. Then, at least for a "typical" $\lambda$, the set $A_{\lambda}$ has an "overlap." Along with the set $A_{\lambda}$ we consider the natural measure $\nu_{\lambda}$ supported on it, obtained by assigning equal probabilities $\frac{1}{2}$ to each of the maps in the IFS. These measures are the complex analogs of infinite Bernoulli convolutions, much studied since the 1930's, see [19] for a survey. We extend classical results of Erdős [8] and Garsia [11] to obtain two classes of complex algebraic integers that give rise to singular and absolutely continuous measures $\nu_{\lambda}$ respectively.

Our final theme is the so-called "typical $\lambda$ " or "almost sure results." Since the sets $A_{\lambda}$ and the measures $\nu_{\lambda}$, for $\lambda \in \mathcal{M}$, are so difficult to study, we attempt to establish dimension formulae, etc., for almost every $\lambda$ with respect to the area measure $\mathcal{L}_{2}$. This line of research has been very active in the last 10 years or so, see, e.g., 24, 28, 22, 18]. They key to obtain results of this type is a certain transversality condition, which in our case means the absence of double zeros for power series with $\{0, \pm 1\}$ coefficients. The extension to the complex plane was started in [29], but it was hampered by the lack of effective estimates of double zeros. Recently, we became aware of such estimates in [3], which yield definitive "typical $\lambda$ " statements for $\lambda \in \mathcal{M}$, with $|\lambda|<1 / \sqrt{2}$ (when $\nu_{\lambda}$ is a priori singular), and partial results for $|\lambda|>1 / \sqrt{2}$ (when $\nu_{\lambda}$ is expected to be absolutely continuous for a typical $\lambda)$.

To conclude the introduction, we note that the sets $A_{\lambda}$ and the measures $\nu_{\lambda}$ arise as "building blocks" when analyzing attractors $E(T, \mathbf{a})$ of IFS $\{T \mathbf{x}, T \mathbf{x}+\mathbf{a}\}$ where $T$ is a linear transformation in $\mathbb{R}^{d}$ and $\mathbf{a}$ is a generic non-zero vector. For instance, if $T$ has a complex eigenvalue $\lambda$, then the projection of $E(T, \mathbf{a})$ onto the $T$-invariant real plane corresponding to $\lambda$ is an affine copy of $A_{\lambda}$. This served as an additional motivation for our study.

\section{Statement of Results}

2.1. Structure of $\mathcal{M}$. We begin with some basic facts about $A_{\lambda}$ and $\mathcal{M}$; they are not difficult and may be found in [4, 2]. Then we briefly mention 
more recent results; see [1] for more information. We have

$$
A_{\lambda}=\left\{\sum_{n=0}^{\infty} a_{n} \lambda^{n}: a_{n} \in\{-1,1\}\right\} .
$$

Since $\lambda \in \mathcal{M}$ if and only if $\left(\lambda A_{\lambda}+1\right) \cap\left(\lambda A_{\lambda}-1\right) \neq \emptyset$, it follows that $\mathcal{M}$ is the set of zeros of $\{0, \pm 1\}$ power series:

$$
\mathcal{M}=\left\{\lambda \in \mathbb{D}: \exists\left\{a_{k}\right\}_{k=1}^{\infty}, a_{k} \in\{-1,0,1\}, 1+\sum_{k=1}^{\infty} a_{k} \lambda^{k}=0\right\} .
$$

From (2.2) it follows that $\mathcal{M}$ is relatively closed in the unit disc $\mathbb{D}$. The following provides easy estimates for $\mathcal{M}$ in terms of $|\lambda|$ :

$$
\left\{\lambda \in \mathbb{D}:|\lambda| \geq 2^{-1 / 2}\right\} \subset \mathcal{M} \subset\{\lambda \in \mathbb{D}:|\lambda| \geq 1 / 2\} .
$$

In their very interesting paper, Odlyzko and Poonen [17] investigated the set of zeros of power series with coefficients 0,1 . Although [17 does not have an immediate application to $\mathcal{M}$ and the family $\left\{A_{\lambda}\right\}$, it turned out to be very useful in this area. In particular, using the ideas of [17], Bousch [5] proved that $\mathcal{M}$ is connected and locally connected (in contrast with the classical Mandelbrot set, for which local connectedness is a famous open problem). Interesting new results were recently obtained by Bandt [1]. In particular, he gave a rigorous computer-assisted proof that $\mathcal{M}$ has "holes" (i.e., $\mathbb{D} \backslash \mathcal{M}$ has more than one component).

A peculiar feature of the set $\mathcal{M}$ is the "antenna" (or "spike") on the positive real axis, from 0.5 to about .67, see 田, 29, 1]. More precisely, there is a line segment $J=[.5, \alpha]$, with $\alpha \approx .67$, such that $J \not \subset \operatorname{clos}(\mathcal{M} \backslash \mathbb{R}$ ). (Of course, there is a symmetric "antenna," on the negative real axis. The set $\mathcal{M}$ is clearly symmetric with respect to both axes, so we will always confine ourselves to the first quarter of the plane.) By (2.3), the "interesting part" of the set $\mathcal{M}$ lies in the disc $\left\{\lambda \in \mathbb{D}:|\lambda| \leq 2^{-1 / 2}\right\}$. Bandt [1]] conjectured that the set $\mathcal{M} \backslash \mathbb{R}$ is contained in the closure of $\operatorname{int}(\mathcal{M})$. In the next theorem we prove a partial result in this direction.

Notation. Denote by $B_{r}\left(z_{0}\right)$ the open disc of radius $r$ centered at $z_{0}$ and write $B_{r}:=B_{r}(0)$.

Theorem 2.1. Let $H=\left\{\lambda \in B_{1 / \sqrt{2}}: \operatorname{Re}(\lambda)>0, \operatorname{Im}(\lambda)>0\right\} \backslash B_{2 / 3}(1 / 3)$; then

$$
\mathcal{M} \cap \operatorname{int}(H) \subset \operatorname{clos}(\operatorname{int}(\mathcal{M}))
$$


The set $H$ is shown in Figure 2. One can check that

$$
H=\left\{\lambda \in \mathbb{D}: 1 / 3 \leq|\lambda|^{2} \leq 1 / 2,0 \leq \operatorname{Re}(\lambda) \leq\left(3|\lambda|^{2}-1\right) / 2\right\} .
$$

The right-most point in $H$ is $\frac{1}{4}+i \frac{\sqrt{7}}{4}$ (incidentally, the corresponding set $A_{\lambda}$ is known as the "tame twindragon"). Figure 3 (made by C. Bandt) shows the set $\mathcal{M} \cap\{\lambda: 0 \leq \operatorname{Re}(\lambda) \leq 1 / 4\}$, which is slightly larger than $\mathcal{M} \cap H$.

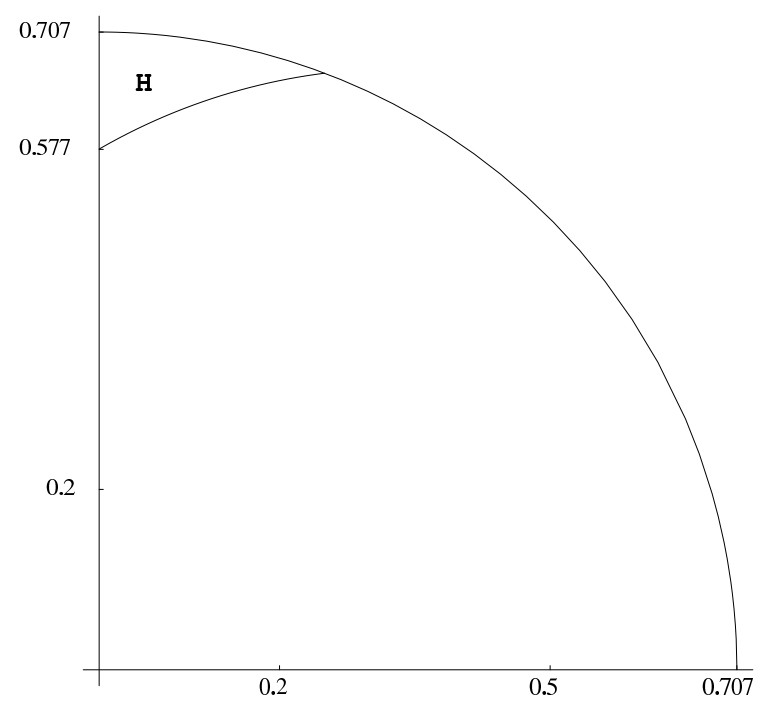

Figure 2. The set $H$

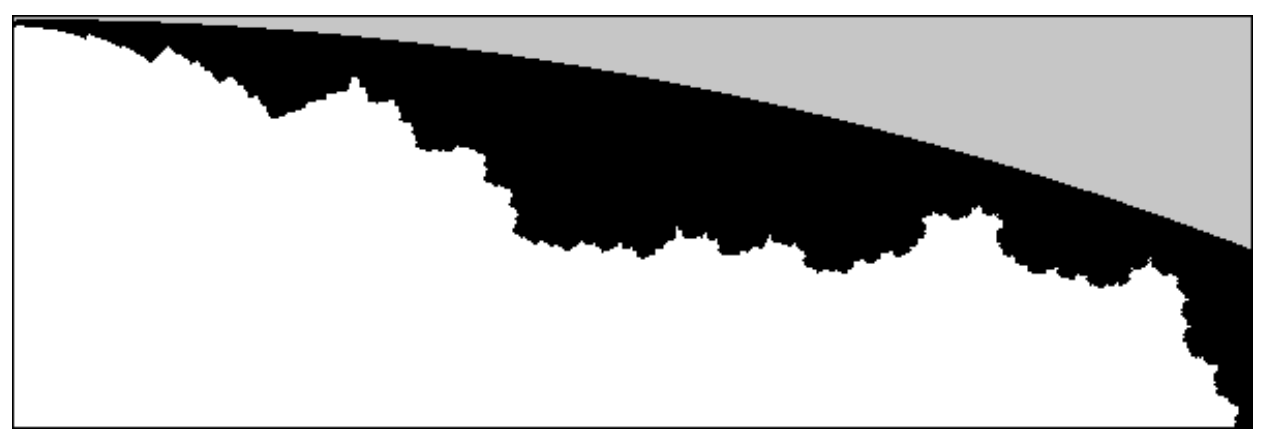

Figure 3. A part of the set $\mathcal{M}$ 
Our method yields many concrete examples of interior points in $\mathcal{M} \cap$ $B_{1 / \sqrt{2}}$. Let $\mathcal{M}_{0}$ be the set of zeros of polynomials with coefficients in $\{0, \pm 1\}$, that is,

$$
\mathcal{M}_{0}:=\left\{\lambda \in \mathbb{D}: \exists\left\{a_{k}\right\}_{k=1}^{n}, a_{k} \in\{-1,0,1\}, 1+\sum_{k=1}^{n} a_{k} \lambda^{k}=0\right\} .
$$

We prove that all points in $\mathcal{M}_{0} \cap \operatorname{int}(H)$ lie in the interior of $\mathcal{M}$. For example, $\lambda_{0} \approx .141964+.677696 i$, a zero of the polynomial $1+z^{2}+z^{3}-z^{4}-z^{5}+z^{7}$, has this property, and we check that its neighborhood of radius $2 \cdot 10^{-3}$ lies in $\mathcal{M}$.

We should note that our method is rather similar to that of Indlekofer, Járai and Kátai, who gave a computer-assisted proof that $\mathcal{M}$ contains $e^{-2 \pi i / 5} / \sqrt{2}$ in its interior [14, p. 423], but there are significant differences as well, see the discussion at the end of Section 3 .

2.2. Complex Bernoulli convolutions. Let $\nu_{\lambda}$ be the distribution of the random series $\sum_{0}^{\infty} \pm \lambda^{n}$ where the signs are chosen independently with probabilities $\left\{\frac{1}{2}, \frac{1}{2}\right\}$. It is the infinite convolution product of $\frac{1}{2}\left(\delta_{-\lambda^{n}}+\delta_{\lambda^{n}}\right)$, a probability measure on the plane. Alternatively, $\nu_{\lambda}$ may be defined as the unique probability measure such that

$$
\nu_{\lambda}=\frac{1}{2}\left(\nu_{\lambda} \circ S_{1}^{-1}+\nu_{\lambda} \circ S_{2}^{-1}\right),
$$

where $S_{1}(z)=\lambda z+1$ and $S_{2}(z)=\lambda z-1$, see 113. Thus, $\nu_{\lambda}$ is a self-similar measure, and by the Law of Pure Type it is either singular or absolutely continuous with respect to the Lebesgue measure $\mathcal{L}_{2}$ (see [19 for a short proof). Observe that $A_{\lambda}$ is precisely the compact support of $\nu_{\lambda}$. It is obvious that $\mathcal{L}_{2}\left(A_{\lambda}\right)=0$ for $|\lambda|<2^{-1 / 2}$, so $\nu_{\lambda}$ is singular. The question "for which $\lambda$, with $|\lambda| \geq 2^{-1 / 2}$, is $\nu_{\lambda}$ absolutely continuous ?" is the complex analog of a well-known problem, studied since the 1930's and still not completely solved (see 19] for a survey).

Definition 2.2. An algebraic integer $\alpha>1$ is a Pisot number (or PVnumber), if all its Galois conjugates (i.e., other roots of the minimal polynomial) are less than one in modulus.

A non-real algebraic integer $\theta$, with $|\theta|>1$, is called a complex Pisot number if all its Galois conjugates, except $\bar{\theta}$, are less than one in modulus.

Below singular/absolutely continuous (a.c.) is always understood with respect to the planar Lebesgue measure $\mathcal{L}_{2}$, unless stated otherwise. 
The following theorem extends the result of Erdös [8], who proved that $\nu_{\lambda}$ is singular with respect to $\mathcal{L}_{1}$ for all real $\lambda \in\left(\frac{1}{2}, 1\right)$ such that $1 / \lambda$ is a Pisot number. (It is an open problem whether this condition is also necessary, i.e., whether $\nu_{\lambda}$ is a.c. with respect to $\mathcal{L}_{1}$ for all real $\lambda \in\left(\frac{1}{2}, 1\right)$ other than reciprocals of Pisot numbers.)

Theorem 2.3. If $\theta$ is a complex Pisot number and $1<|\theta|<\sqrt{2}$, then $\nu_{\lambda}$ is singular for $\lambda=1 / \theta$.

Remarks. 1. If $|\theta|>\sqrt{2}$, then $\nu_{\lambda}$, for $\lambda=1 / \theta$, is singular for the trivial reason that $\mathcal{L}_{2}\left(A_{\lambda}\right)=0$.

2. It is well-known (Siegel [26], see also [6]) that the smallest real Pisot number is $\alpha_{0} \approx 1.3247$, the positive zero of $z^{3}-z-1$. Chamfy $[7]$ found the smallest in modulus complex Pisot numbers: their modulus is $\sqrt{\alpha_{0}} \approx 1.1509$, with either $z^{3}-z^{2}+1$ or $z^{6}-z^{2}+1$ as a minimal polymial. Garth 12] found a list of all sufficiently small complex Pisot numbers. The reciprocals of ten smallest complex Pisot numbers in the first quarter of the plane are shown in Figure 4.

3. Let $S_{1}, S_{2}$ denote the sets of real and complex Pisot numbers respectively. There are no quadratic numbers in $S_{2} \cap B_{\sqrt{2}}$. Among the cubic numbers in $S_{2} \cap B_{\sqrt{2}}$ are zeros of $z^{3}-z^{2}+z+1, z^{3}+z+1$, and $z^{3}-z^{2}+1$. The set $S_{2} \cap B_{\sqrt{2}}$ also includes all numbers $\pm i \sqrt{\alpha}$ where $\alpha \in(1,2)$ is a real Pisot number.

4. The set $S_{2} \cup \pm S_{1}$ is closed, see [6, 9.2].

Definition 2.4. We say that an algebraic integer $\theta$, with $|\theta|>1$, is a Garsia number if all its Galois conjugates are greater than one in modulus. A complex Garsia number will mean a non-real Garsia number.

The following theorem extends the result of Garsia [11], who proved that $\nu_{\lambda}$ is a.c. with respect to $\mathcal{L}_{1}$, with a bounded density, for all real $\lambda \in\left(\frac{1}{2}, 1\right)$ such that $1 / \lambda$ is a Garsia number, whose minimal polynomial has constant term \pm 2 .

Theorem 2.5. If $\theta$ is a complex Garsia number and the minimal polynomial for $\theta$ has constant term \pm 2 , then $\nu_{\lambda}$, for $\lambda=1 / \theta$, is a.c. with a bounded density.

Remarks. 1. The conditions on $\theta$ imply that $1<|\theta| \leq \sqrt{2}$, since $\bar{\theta}$ is always a conjugate and the product of all zeros equals \pm 2 . The equality $|\theta|=\sqrt{2}$ implies that $\theta$ is quadratic. There are exactly ten such complex 


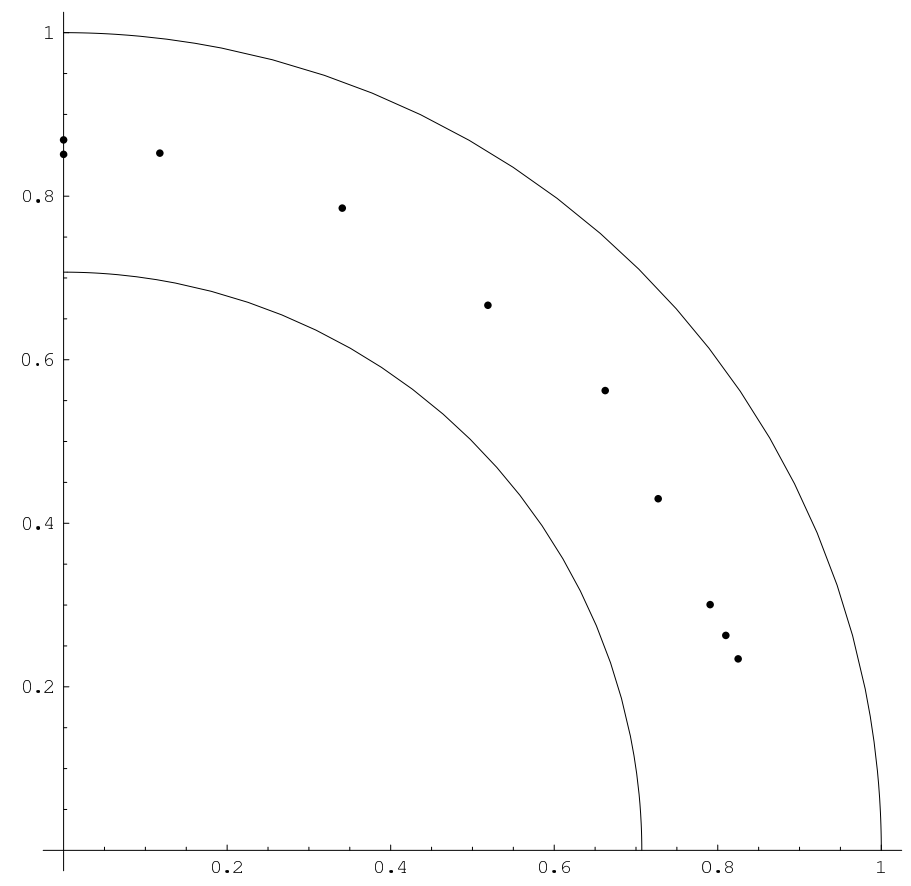

Figure 4. Complex Pisot numbers

Garsia numbers: $\pm i \sqrt{2}, \pm 1 \pm i, \pm \frac{1}{2} \pm i \frac{\sqrt{7}}{2}$, which yield three essentially different measures $\nu_{\lambda}$. The compact supports $A_{\lambda}$ of these measures are wellknown as "reptiles" that tile the plane periodically: $A_{\lambda}$ is a rectangle for $\lambda=i \cdot 2^{-1 / 2}$, the "twindragon" for $\lambda=\frac{1}{2}+i \frac{1}{2}$, and the "tame twindragon" for $\lambda=\frac{1}{4}+i \frac{\sqrt{7}}{4}$. The measure $\nu_{\lambda}$ in each of these cases is just the normalized Lebesgue measure restricted to $A_{\lambda}$.

2. As in 11], it is easy to see that if $q$ is a polynomial with integer coefficients, having the leading coefficient one and the constant term \pm 2 , with all roots satisfying $\left|\theta_{j}\right| \in[1,2]$, then every non-real root $\theta$ of $q$ is a complex Garsia number. (Indeed, consider the minimal polynomial $p$ of $\theta$, of degree $\ell$. It cannot have roots of modulus one, since otherwise $p(z)$ and $z^{\ell} p(1 / z)$ would have a common zero, hence would have the same set of zeros, 
which is a contradiction.) Thus, all non-real roots of the polynomials given in [11, 1.8] are complex Garsia numbers. We get a countable set of such numbers from the polynomials $x^{m+n}-x^{n}-2$, with $\max \{m, n\} \geq 2$. It is easy to see that such a polynomial has all roots $\left|\theta_{j}\right| \in[1,2]$ and at most two roots are real.

3. Garsia numbers whose minimal polynomial has constant term $\pm m$, for $m>2$, occur in a similar context as well-their reciprocals $\lambda$ have the property that the random series $\sum_{j=0}^{\infty} a_{j} \lambda^{j}$ has a.c. distribution, where $a_{j}$ are random and independent and take values in $\{0,1, \ldots, m-1\}$ (or any other set of $m$ equidistant digits) with equal probabilities. (If $\lambda$ is real, then the distribution is a.c. with respect to $\mathcal{L}_{1}$; otherwise it is a.c. with respect to $\mathcal{L}_{2}$.)

2.3. The sets $A_{\lambda}$ for $|\lambda| \geq 1 / \sqrt{2}$. If the measure $\nu_{\lambda}$ is absolutely continuous, then, of course, its support $A_{\lambda}$ has positive area, and if the density of $\nu_{\lambda}$ is continuous, then $A_{\lambda}$ has non-empty interior. However, the converse is false. In particular, for all $\lambda \in\left[i \cdot 2^{-1 / 2}, i\right)$ the set $A_{\lambda}$ is a rectangle, and so obviously has non-empty interior, whereas infinitely many $\lambda$ in this segment on the imaginary axis are reciprocals of complex Pisot numbers and so $\nu_{\lambda}$ is singular. It is plausible that $A_{\lambda}$ has non-empty interior for all non-real $\lambda$ with $1 / \sqrt{2}<|\lambda|<1$. Some elementary results in this direction are contained in the following proposition. Part (ii) is a straightfoward extension of [14, p. 424].

Proposition 2.6. (i) $A_{\lambda}$ has non-empty interior for all $\lambda$ in

$$
\begin{aligned}
\Omega & :=\{\lambda \in \mathbb{D}: \operatorname{Re}(\lambda) \geq 0, \operatorname{Im}(\lambda) \geq 0\} \backslash B_{\sqrt{3} / 2}(1 / 2) \\
& =\left\{\lambda \in \mathbb{D}: 0 \leq \operatorname{Re}(\lambda) \leq|\lambda|^{2}-(1 / 2)\right\} .
\end{aligned}
$$

(ii) If $\lambda=r e^{\pi i m / n}$, with $r \in\left[2^{-1 / n}, 1\right)$, where $G C D(m, n)=1$, then $A_{\lambda}$ is $a(2 n)$-gon having all angles equal to $\frac{\pi(n-1)}{n}$, so it has non-empty interior. If $\lambda=r e^{2 \pi i m /(2 n+1)}$, with $r \in\left[2^{-1 /(2 n+1)}, 1\right)$, where $G C D(m, 2 n+1)=1$, then $A_{\lambda}$ is a $(4 n+2)$-gon having all angles equal to $\frac{\pi(2 n)}{2 n+1}$, so it has non-empty interior.

For additional results on $A_{\lambda}$ with non-empty interior, see Theorem 2.10(ii) below.

For completeness, we mention what happens if $|\lambda|=1 / \sqrt{2}$, although we do not have anything new in this case. Then the similarity dimension of $A_{\lambda}$ equals 2 , so $\mathcal{L}_{2}\left(A_{\lambda}\right)>0$ if and only if $A_{\lambda}$ has non-empty interior 
[25]. It is conjectured that this happens only for quadratic $\lambda$ in Remark 1 to Theorem 2.5; this seems to be a difficult problem. Some progress on a related conjecture was made in [14].

2.4. Results for a "typical" $\lambda$. By "typical" we mean almost every (a.e.) with respect to the Lebesgue measure $\mathcal{L}_{2}$. We do not review the history of this development, but refer the reader to [24, 28, 22, 29, 18, 19]. A key technical element needed for these results is a "transversality condition." Let

$$
\mathcal{B}_{\{0, \pm 1\}}=\left\{1+\sum_{k=1}^{\infty} a_{k} z^{k}: a_{k} \in\{-1,0,1\}\right\} .
$$

Thus, $\mathcal{M}=\left\{\lambda \in \mathbb{D}: \exists f \in \mathcal{B}_{\{0, \pm 1\}}, f(\lambda)=0\right\}$. Consider the set of double zeros

$$
\widetilde{\mathcal{M}}=\left\{\lambda \in \mathbb{D}: \exists f \in \mathcal{B}_{\{0, \pm 1\}}, f(\lambda)=f^{\prime}(\lambda)=0\right\} .
$$

We say that $\mathbb{D} \backslash \widetilde{\mathcal{M}}$ is the region of transversality for $\mathcal{B}_{\{0, \pm 1\}}$. The following result was established in 1998 (and a preprint was available since 1995), although it was not explicitly stated in this form.

Proposition 2.7. [3] A power series of the form $1+\sum_{n=1}^{\infty} a_{n} z^{n}$, with $a_{n} \in$ $[-1,1]$, cannot have a non-real double zero of modulus less than $2 \cdot 5^{-5 / 8} \approx$ .73143 .

This yields the desired transversality on $\mathcal{M} \cap B_{1 / \sqrt{2}}$ and in a thin annulus outside $B_{1 / \sqrt{2}}$. Below we collect the consequences for $A_{\lambda}$ and $\nu_{\lambda}$. Let

$$
s(\lambda)=\frac{\log 2}{-\log |\lambda|}
$$

denote the similarity dimension of the set $A_{\lambda}$.

Theorem 2.8. We have $\operatorname{dim}_{H}\left(A_{\lambda}\right)=s(\lambda)$ for a.e. $\lambda \in \mathcal{M} \cap B_{1 / \sqrt{2}}$. Moreover, for any $0<r<R<1 / \sqrt{2}$,

$$
\operatorname{dim}_{H}\left\{\lambda \in \mathcal{M}: r<|\lambda|<R, \operatorname{dim}_{H} A_{\lambda}<s(\lambda)\right\} \leq \frac{\log 2}{-\log R}<2 .
$$

This follows from [29] and [18, Thm 8.2], in view of Proposition 2.7.

Remarks. 1. Since $A_{\lambda}$ is self-similar, its Hausdorff, Minkowski, and packing dimensions are all equal [9].

2. It is well-known that $\operatorname{dim}_{H}\left(A_{\lambda}\right) \leq s(\lambda)$ for all $\lambda$, see, e.g., [10, 9.6].

3. It is proved in [29, Prop 2.3] that $\operatorname{dim}_{H}\left(A_{\lambda}\right)<s(\lambda)$ for a dense set of $\lambda \in \mathcal{M} \cap B_{1 / \sqrt{2}}$. In fact, this holds for all $\lambda \in \mathcal{M}_{0} \cap B_{1 / \sqrt{2}}$. (Recall 
that $\mathcal{M}_{0}$ is the set of zeros of polynomials with $\{0, \pm 1\}$ coefficients.) It is a challenging open problem whether there exist $\lambda \notin \mathcal{M}_{0} \cap B_{1 / \sqrt{2}}$ such that $\operatorname{dim}_{H}\left(A_{\lambda}\right)<s(\lambda)$.

Denote by $\mathcal{H}^{s}$ and $\mathcal{P}^{s}$ the $s$-dimensional Hausdorff and packing measure respectively (see, e.g., 10] for definitions). The next theorem says that for a typical $\lambda \in \mathcal{M} \cap B_{1 / \sqrt{2}}$, the $s(\lambda)$-dimensional Hausdorff measure of $A_{\lambda}$ is zero, whereas the packing measure $\mathcal{P}^{s(\lambda)}\left(A_{\lambda}\right)$ is positive and finite.

Theorem 2.9. (i) We have $\mathcal{H}^{s(\lambda)}\left(A_{\lambda}\right)=0$ for a.e. $\lambda \in \mathcal{M} \cap B_{1 / \sqrt{2}}$.

(ii) We have $\mathcal{P}^{s(\lambda)}\left(A_{\lambda}\right)<\infty$ for all $\lambda$ and $\mathcal{P}^{s(\lambda)}\left(A_{\lambda}\right)>0$ for a.e. $\lambda \in$ $\mathcal{M} \cap B_{1 / \sqrt{2}}$. Moreover, for any $0<r<R<1 / \sqrt{2}$,

$$
\operatorname{dim}_{H}\left\{\lambda \in \mathcal{M}: r<|\lambda|<R, \mathcal{P}^{s(\lambda)}\left(A_{\lambda}\right)=0\right\} \leq \frac{\log 2}{-\log R}<2 .
$$

(iii) For a.e. $\lambda \in \mathcal{M} \cap B_{1 / \sqrt{2}}$ the measure $\nu_{\lambda}$ is equivalent to $\left.\mathcal{P}^{s(\lambda)}\right|_{A_{\lambda}}$.

This is proved using the methods of [20]; again, transversality (Proposition 2.7) is essential. The needed modifications are fairly straightforward; they are left to the reader.

Remarks. 1. Schief [25] proved that a self-similar set has zero Hausdorff measure in its similarity dimension if and only if the open set condition fails. (In our case the validity of the open set condition means, by definition, that there is a non-empty open set $U \subset \mathbb{C}$ such that its images $\lambda U \pm 1$ lie in $U$ and do not intersect.) It is sometimes considered that "overlapping" is synonymous with the failure of the open set condition.

2. There is a "topological" version of the Hausdorff dimension and Hausdorff measure result (but not of the packing measure result). In fact, there is a dense $G_{\delta}$ subset $\Phi \subset \mathcal{M} \cap B_{1 / \sqrt{2}}$ such that $\operatorname{dim}_{H}\left(A_{\lambda}\right)=s(\lambda)$, with $\mathcal{H}^{s(\lambda)}\left(A_{\lambda}\right)=0$, for all $\lambda \in \Phi$. The dimension formula follows from the lower semi-continuity of Hausdorff dimension as a function of parameter [16], as in [27, Th.2.3]. Zero Hausdorff measure follows from [20].

Next we turn to the case $|\lambda|>1 / \sqrt{2}$ again. Then the similarity dimension of $A_{\lambda}$ exceeds 2. It is conjectured that for a.e. such $\lambda$ the measure $\nu_{\lambda}$ is absolutely continuous and the set $A_{\lambda}$ has positive area. The following theorem is a partial result in this direction.

Theorem 2.10. (i) The measure $\nu_{\lambda}$ is a.c. with a density in $L^{2}\left(\mathbb{R}^{2}\right)$, hence $\mathcal{L}_{2}\left(A_{\lambda}\right)>0$, for a.e. $\lambda \in\left\{z \in \mathbb{C}: 2^{-1 / 2} \leq|z| \leq 2 \cdot 5^{-5 / 8}\right\}$. (Note that 
$2^{-1 / 2} \approx .7071067,2 \cdot 5^{-5 / 8} \approx .7314316$.) Moreover, for any $2^{-1 / 2}<r<$ $R<2 \cdot 5^{-5 / 8}$,

$$
\operatorname{dim}_{H}\left\{\lambda \in \mathbb{C}: r<|\lambda|<R, d \nu_{\lambda} / d x \notin L^{2}\left(\mathbb{R}^{2}\right)\right\} \leq 4-\frac{\log 2}{-\log r} .
$$

(ii) The measure $\nu_{\lambda}$ is a.c. with a continuous density, hence $A_{\lambda}$ has nonempty interior, for a.e. $\lambda$ such that

$$
|\lambda| \in \bigcup_{k=2}^{\infty}\left(2^{-1 /(2 k)},\left(2 \cdot 5^{-5 / 8}\right)^{1 / k}\right)
$$

in particular, for a.e. $\lambda \in \mathbb{D}$ with $|\lambda|>2^{-1 / 20} \approx .9659363$.

Part (i) follows from [29] and [18, Thm 8.2], in view of Proposition 2.7. Part (ii) easily follows from (i); we indicate the proof in Section 5.

Remark. Although with the methods of [21, 22] we can somewhat increase the region where the statements in the last theorem hold, unfortunately, we are not able to "cover" the whole annulus $1 / \sqrt{2}<|\lambda|<1$.

\section{INTERIOR POINTS OF $\mathcal{M}$}

In this section we prove Theorem 2.1. The proof is based on several lemmas. They are all quite simple, but one of them requires some calculations and its proof is postponed to the end of the section. (We emphasize, however, that our proof does not rely on computer.) Let

$$
A_{\lambda}\{-1,0,1\}=\left\{\sum_{n=0}^{\infty} a_{n} \lambda^{n}: a_{n} \in\{-1,0,1\}\right\} .
$$

Observe that $A_{\lambda}\{-1,0,1\}$ is the attractor of the IFS $\{\mathbb{C} ; \lambda z-1, \lambda z, \lambda z+1\}$. The following lemma is standard, see e.g. [14, Lemma 7].

Lemma 3.1. If $F \subset \mathbb{C}$ is compact, $\lambda \in \mathbb{C}$, and

$$
F \subset \lambda F \cup(\lambda F-1) \cup(\lambda F+1),
$$

then $F \subset A_{\lambda}\{-1,0,1\}$.

Lemma 3.2. If $F \subset A_{\lambda}\{-1,0,1\}$ and there exists a finite sequence $\left\{a_{k}\right\}_{k=1}^{n}$, $a_{k} \in\{-1,0,1\}$, such that

$$
1+\sum_{k=1}^{n} a_{k} \lambda^{k} \in \lambda^{n+1} F,
$$

then $\lambda \in \mathcal{M}$. 
Proof. By (3.1), every point in $\lambda^{n+1} F \subset \lambda^{n+1} A_{\lambda}\{-1,0,1\}$ can be written as $\sum_{k=n+1}^{\infty} b_{k} \lambda^{k}$, with coefficients $b_{k}$ in $\{-1,0,1\}$. Thus, $\lambda \in \mathcal{M}$ by (2.2).

Let $R_{a, b} \subset \mathbb{C}$ denote the rectangle centered at the origin, with the vertices at $\pm a \pm i b$.

Lemma 3.3. For any $\lambda \in H$, there exist $a \geq 1$ and $b \geq 0.5$ such that

$$
R_{a, b} \subset \lambda R_{a, b} \cup\left(\lambda R_{a, b}-1\right) \cup\left(\lambda R_{a, b}+1\right) .
$$

The proof of this lemma is given at the end of the section. Roughly speaking, the reason it works (at least in some region) is that for $\lambda \in\left[i 3^{-1 / 2}, i\right)$, the attractor $A_{\lambda}\{-1,0,1\}$ is exactly a rectangle $R_{a, b}$ for appropriate $a$ and $b$. Recall that $\mathcal{M}_{0}$ denotes the set of zeros of polynomials with coefficients in $\{0, \pm 1\}$. The following lemma is standard.

Lemma 3.4. $\mathcal{M}=\operatorname{clos}\left(\mathcal{M}_{0}\right) \cap \mathbb{D}$.

Proof. Let $\lambda \in \mathcal{M}$. We have $1+\sum_{k=1}^{\infty} a_{k} \lambda^{k}=0$ for some $a_{k} \in\{-1,0,1\}$. One can apply Rouché's Theorem to show that for any $\varepsilon>0$ there exists $n$ such that the polynomial $1+\sum_{k=1}^{n} a_{k} z^{k}$ has a zero in $B_{\varepsilon}(\lambda)$.

Proof of Theorem 2.1. It follows from Lemma 3.1 and Lemma 3.3 that

$$
F:=B_{0.5}(0) \subset A_{\lambda}\{-1,0,1\}
$$

for all $\lambda \in H$. In view of Lemma 3.4, it is enough to show that any $\lambda_{0} \in \operatorname{int}(H) \cap \mathcal{M}_{0}$ is an interior point of $\mathcal{M}$. Since $\lambda_{0} \in \mathcal{M}_{0}$, there exist $a_{1}, \ldots, a_{n} \in\{-1,0$,$\} such that$

$$
p\left(\lambda_{0}\right)=0, \quad \text { where } p(z)=1+\sum_{k=1}^{n} a_{k} z^{k} .
$$

We can find $\delta>0$ such that $B_{\delta}\left(\lambda_{0}\right) \subset H$ and, by the continuity of $p$,

$$
\left|\lambda-\lambda_{0}\right|<\delta \Rightarrow|p(\lambda)|=\left|p(\lambda)-p\left(\lambda_{0}\right)\right| \leq 0.5|\lambda|^{n+1} .
$$

Then for all $\lambda \in B_{\delta}\left(\lambda_{0}\right)$ we have $p(\lambda) \in \lambda^{n+1} F$, hence $\lambda \in \mathcal{M}$ by Lemma 3.2.

Example. It is not difficult to find specific elements of $\mathcal{M}_{0}$ in $\operatorname{int}(H)$. Using Bandt's algorithm for drawing the set $\mathcal{M}$, for any "black" point $\lambda_{1}$ in Figure 1 , we can find a polynomial $p$ with coefficients in $\{0, \pm 1\}$ such that $\left|p\left(\lambda_{1}\right)\right|$ is small. Then $p$ is likely to have a zero near $\lambda_{1}$.

For example, we may take $\lambda_{1}=.14+.68 i \in \operatorname{int}(H)$ and the polynomial $p(z)=1+z^{2}+z^{3}-z^{4}-z^{5}+z^{7}$ (found with the help of Bandt's algorithm), 
which has a zero $\lambda_{0} \approx 0.141964+0.677696 i$. From the proof of Theorem 2.1 it follows that if $\lambda \in \operatorname{int}(H)$ and $\left|p(\lambda)-p\left(\lambda_{0}\right)\right| \leq 0.5|\lambda|^{8}$, then $\lambda \in \mathcal{M}$. We can estimate, assuming that $|\lambda| \leq 2^{-1 / 2}$ :

$$
\begin{aligned}
\left|p(\lambda)-p\left(\lambda_{0}\right)\right| & \leq \sum_{k=1}^{7}\left|\lambda^{k}-\lambda_{0}^{k}\right| \\
& =\left|\lambda-\lambda_{0}\right| \sum_{k=1}^{7}\left|\lambda^{k-1}+\lambda \cdot \lambda_{0}^{k-2}+\cdots+\lambda_{0}^{k-1}\right| \\
& \leq\left|\lambda-\lambda_{0}\right| \sum_{k=1}^{\infty} k \cdot 2^{-(k-1) / 2} \\
& =\left|\lambda-\lambda_{0}\right| \cdot\left(1-2^{-1 / 2}\right)^{-2} .
\end{aligned}
$$

It follows that the disc of radius $2 \cdot 10^{-3}$ centered at $\lambda_{0}$ lies in $\mathcal{M}$. (Indeed, $\left|\lambda_{0}\right|>.692$, so $|\lambda|>.69$ in this disc, and $.69^{8}\left(1-2^{-1 / 2}\right)^{2} / 2>2 \cdot 10^{-3}$.)

Proof of Lemma 3.9. Let $R:=R_{a, b}$ be the rectangle with vertices $\pm a \pm i b$. We assume right away that $a \geq b$ and $a>1$. Let $\lambda=\xi+i \eta$. We assume that $\xi, \eta>0,|\lambda|^{2} \geq \frac{1}{3}$ and $\xi \leq|\lambda|^{2}$ (this is certainly true in the set $H$ ). The condition $R \subset \lambda R \cup(\lambda R-1) \cup(\lambda R+1)$ is equivalent to

$$
\lambda^{-1} R \subset R \cup\left(R-\lambda^{-1}\right) \cup\left(R+\lambda^{-1}\right) .
$$

Figure 5 will help us write down sufficient conditions for (3.4); it shows the case of $\lambda=0.1+0.68 i, a=1.35$, and $b=0.78$. We claim that the following conditions imply (3.4):

(C1) Overlapping: for $R$ to overlap $R \pm \frac{1}{\lambda}$ we need that $|\operatorname{Im}(1 / \lambda)|<2 b$, or equivalently,

$$
|\lambda|^{2} \geq \frac{\eta}{2 b}
$$

(C2) Covering the short sides: we want the vertices $\frac{a \pm i b}{\xi+i \eta}$ of $\frac{1}{\lambda} R$ to lie in $R+\frac{1}{\lambda}$; then by symmetry, the vertices $\frac{-a \pm i b}{\xi+i \eta}$ will be in $R-\frac{1}{\lambda}$. The conditions are:

$$
\begin{aligned}
& -a+\operatorname{Re}(1 / \lambda) \leq \operatorname{Re}[(a \pm i b) / \lambda] \leq a+\operatorname{Re}(1 / \lambda), \\
& -b+\operatorname{Im}(1 / \lambda) \leq \operatorname{Im}[(a \pm i b) / \lambda] \leq b+\operatorname{Im}(1 / \lambda) .
\end{aligned}
$$

(C3) Covering the long sides: we want the upper intersection point of $\partial(\lambda R)$ and $\partial(\lambda R+1)$ to be above or on the line $y=b$. Then by symmetry the same is true for the intersection point of $\partial(\lambda R)$ and $\partial(\lambda R-1)$, and the upper side of $R$ is covered. Again by symmetry, the lower side will be 


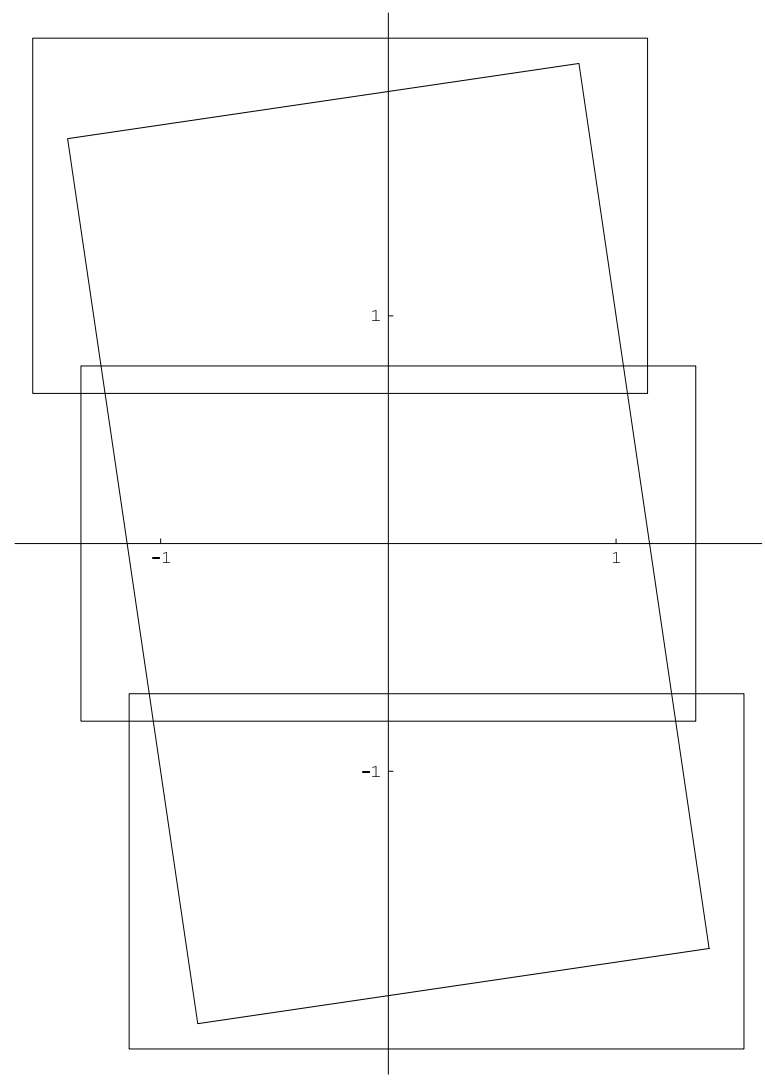

FiguRE 5. $\quad \lambda^{-1} R_{a, b} \subset R_{a, b} \cup\left(R_{a, b}-\lambda^{-1}\right) \cup\left(R_{a, b}+\lambda^{-1}\right)$

covered as well. With the help of Figure 5 we can see that this intersection point is $\lambda(a+i(b+\operatorname{Im}(1 / \lambda))$, so we get the condition

$$
\operatorname{Im}(\lambda(a+i(b+\operatorname{Im}(1 / \lambda))) \geq b
$$

After some algebraic manipulations we get the following four inequalities (it turns out that out of the eight inequalities in (3.6), (3.7) four are always true by our assumptions, and two more follow from the remaining two).

(A1) $|\lambda|^{2} \geq \frac{\eta}{2 b}$

(A2) $a \eta+b \xi \geq b+\frac{\xi \eta}{|\lambda|^{2}}$

(A3) $a \xi+b \eta \leq a|\lambda|^{2}+\xi$

(A4) $a \eta+b \xi \leq b|\lambda|^{2}+\eta$. 
We want to find for which $(\xi, \eta)$ there exist $(a, b)$ such that (A1)-(A4) are satisfied. It is natural to look for the solution by setting some inequalities to be equalities.

First, set (A2) and (A3) to be equalities and solve for $a$ and $b$ to obtain

$$
a=1+\frac{\left(|\lambda|^{2}-\xi\right)^{2}}{|\lambda|^{2}\left(1-|\lambda|^{2}\right)}, \quad b=\frac{\eta\left(|\lambda|^{2}-\xi\right)}{|\lambda|^{2}\left(1-|\lambda|^{2}\right)} .
$$

The inequalities (A1) and (A4) reduce to

$$
|\lambda|^{2}-\frac{|\lambda| \sqrt{1-|\lambda|^{2}}}{\sqrt{2}} \leq \xi \leq \frac{3|\lambda|^{2}-1}{2} .
$$

Next, set (A3) and (A4) to be equalities and solve for $a$ and $b$ to obtain

$$
a=\frac{\eta^{2}+\xi\left(|\lambda|^{2}-\xi\right)}{\eta^{2}-\left(|\lambda|^{2}-\xi\right)^{2}}, \quad b=\frac{\eta|\lambda|^{2}}{\eta^{2}-\left(|\lambda|^{2}-\xi\right)^{2}} .
$$

The inequality (A2) reduces to

$$
\xi \leq|\lambda|^{2}-\frac{|\lambda| \sqrt{1-|\lambda|^{2}}}{\sqrt{2}},
$$

and (A1) follows from (A2) under our assumptions, whenever $|\lambda|^{2} \geq \frac{1}{3}$. Combining (3.10), (3.9), (3.12), and (3.11) yields that the desired $a, b$ may be found for all $\lambda=\xi+i \eta$ such that $\frac{1}{3} \leq|\lambda|^{2} \leq \frac{1}{2}$ and $0 \leq \xi \leq \frac{3|\lambda|^{2}-1}{2}$. This is equivalent to $\lambda \in H$. It remains to check the initial assumptions on $a$ and $b$. It is immediate from (3.9) and (3.11) that $a \geq 1$. To estimate $b$, we note that every $\lambda \in H$ satisfies $|\lambda|^{2} \leq \frac{1}{2}$, so $\xi \leq \frac{3|\lambda|^{2}-1}{2} \leq \frac{1}{4}$. On the other hand, $\xi^{2}+\eta^{2}=|\lambda|^{2} \geq \frac{1}{3}$. Thus, by (A1),

$$
b \geq \frac{\eta}{2|\lambda|^{2}} \geq \eta \geq \sqrt{\frac{1}{3}-\xi^{2}} \geq \sqrt{\frac{1}{3}-\frac{1}{16}}=\sqrt{\frac{13}{48}}>\frac{1}{2},
$$

as desired. It remains to check that $a \geq b$. By (A2), $\frac{a}{b} \geq \frac{1-\xi}{\eta}$. But $|\lambda|^{2}=\left(\xi^{2}+\eta^{2}\right) \leq \frac{1}{2}$, hence $\xi+\eta \leq 1$, and so $\frac{a}{b} \geq 1$.

Remarks. 1. One might hope to extend this method to show that the whole set $\mathcal{M}$ is the closure of its interior (as conjectured by Bandt [1]). To do this, one would need to prove that the attractor $A_{\lambda}\{-1,0,1\}$ contains some neighborhood of the origin, with a radius locally uniformly bounded below. It is expected that for $|\lambda|>3^{-1 / 2}$ (with $\lambda$ non-real) the attractor $A_{\lambda}\{-1,0,1\}$ has positive measure and non-empty interior, at least, for a typical $\lambda$, since then the similarity dimension of the IFS is greater than 2 . However, this remains an open problem. 
2. In Lemma 3.1, it is enough that $F$ is covered not just by the images under one iteration of the IFS, but by images under any number of iterations; the conclusion remains the same. Moreover, instead of rectangles, one can consider other simple shapes and attempt to establish the covering property. A similar method was employed in [14]. In that paper the authors used a disc rather than rectangle for the set $F$. They considered $\lambda=e^{-2 \pi i / 5} / \sqrt{2}$ and had to use 5 iterations of the IFS to achieve the needed covering property. This involved drawing $\left(3^{6}-1\right) / 2$ circles and heavy computer use.

\section{The CASE OF $|\lambda| \geq 1 / \sqrt{2}$}

Here we prove Theorems 2.3 and 2.5 and Proposition 2.6.

Proof of Theorem 2.3. This is a straightforward extension of [8], but we provide the argument for completeness. Consider the Fourier transform

$$
\widehat{\nu}_{\lambda}(\xi)=\int_{\mathbb{R}^{2}} e^{i t \cdot \xi} d \nu_{\lambda}(t)
$$

where $\xi, t \in \mathbb{R}^{2}$ and $t \cdot \xi$ is the dot product. Considering $\xi$ and $t$ as complex numbers we have $t \cdot \xi=\operatorname{Re}(t \bar{\xi})$. Then, by the independence of the random series defining $\nu_{\lambda}$,

$$
\widehat{\nu}_{\lambda}(\xi)=\prod_{n=0}^{\infty} \frac{1}{2}\left({\widehat{\lambda^{n}}}^{n+\delta}-\lambda^{n}\right)(\xi)=\prod_{n=0}^{\infty} \cos \left(\operatorname{Re}\left(\lambda^{n} \bar{\xi}\right)\right) .
$$

Let $\theta$ be a complex Pisot number, with $|\theta|^{2} \in(1,2)$. Then $\theta$ has degree at least three, since $|\theta|^{2}=\theta \bar{\theta}$ is not an integer, and the product of all roots of the minimal polynomial is an integer. Let $\theta_{3}, \ldots, \theta_{m}$ be the algebraic (Galois) conjugates of $\theta$ other than $\bar{\theta}$. We know that $\left|\theta_{j}\right|<1$ for $j \geq 3$. Observe that $\theta$ is an algebraic unit, since the constant term of the minimal polynomial $(-1)^{m}|\theta|^{2} \prod_{j=3}^{m} \theta_{j}$ is less than 2 in modulus, so it must be equal to 1 . Since $\theta^{n}+\bar{\theta}^{n}+\sum_{j=3}^{m} \theta_{j}^{n} \in \mathbb{Z}$ for all $n \geq 1$, we have

$$
\operatorname{dist}\left(2 \operatorname{Re}\left(\theta^{n}\right), \mathbb{Z}\right) \leq c \rho^{n}, \quad n \geq 1,
$$

for some $\rho \in(0,1)$ and $c>0$. Recall that $\theta=1 / \lambda$. We have

$$
\widehat{\nu}_{\lambda}\left(2 \pi \bar{\theta}^{N}\right)=\prod_{n=-\infty}^{N} \cos \left(2 \pi \operatorname{Re}\left(\theta^{n}\right)\right)=\prod_{n=1}^{N} \cos \left(2 \pi \operatorname{Re}\left(\theta^{n}\right)\right) \cdot \widehat{\nu}_{\lambda}(2 \pi) .
$$

We claim that $\widehat{\nu}_{\lambda}\left(2 \pi \bar{\theta}^{N}\right) \neq 0$ for $N \in \mathbb{N}$. Indeed, since $\theta$ is an algebraic unit, $\theta^{-1}$ is also an algebraic unit, and therefore, $\theta^{n}$ is an algebraic integer for all $n \in \mathbb{Z}$. Now $2 \operatorname{Re}\left(\theta^{n}\right)=\theta^{n}+\bar{\theta}^{n}$ is also an algebraic integer (as a sum 
of algebraic integers) for all $n \in \mathbb{Z}$, so it cannot be equal $k+\frac{1}{2}$, for some integer $k$. It follows that $\cos \left(2 \pi \operatorname{Re}\left(\theta^{n}\right)\right) \neq 0$ for all $n \in \mathbb{Z}$, which implies the claim.

Now (4.3) and (4.2) imply

$$
\left|\widehat{\nu}_{\lambda}\left(2 \pi \bar{\theta}^{N}\right)\right| \geq \prod_{n=1}^{\infty}\left|\cos \left(c \rho^{n}\right)\right| \cdot\left|\widehat{\nu}_{\lambda}(2 \pi)\right|=: \delta>0,
$$

for all $N \geq 1$. Thus, $\widehat{\nu}_{\lambda}(\xi) \nrightarrow \rightarrow 0$ as $|\xi| \rightarrow \infty$. By the Riemann-Lebesgue Lemma, $\nu_{\lambda}$ is not absolutely continuous, so by the Law of Pure Type, $\nu_{\lambda}$ is singular.

Proof of Theorem 2.5. This is a straightforward extension of [11], but we provide the argument for completeness. For $n \geq 1$ let

$$
\mathcal{A}_{\lambda}^{(n)}:=\left\{\sum_{k=0}^{n-1} a_{k} \lambda^{k}: a_{k}= \pm 1\right\}
$$

We will prove the following two claims:

$$
\# \mathcal{A}_{\lambda}^{(n)}=2^{n} .
$$

(4.5尹 $c>0, \forall n \geq 1,|x-y| \geq c \cdot 2^{-n / 2}$ for all $x, y \in \mathcal{A}_{\lambda}^{(n)}, x \neq y$.

First we deduce the desired statement follows from (4.4) and (4.5). Absolute continuity of $\nu_{\lambda}$ with a bounded density will follow if we can show that

$$
\nu_{\lambda}\left(B_{r}(z)\right) \leq \text { const } \cdot r^{2} \text { for all } z \in \mathbb{C}
$$

for all $r>0$. Let $C_{1}>0$ be such that $A_{\lambda} \subset B_{C_{1}}$. Clearly, it is enough to establish (4.6) for $r=C_{1}|\lambda|^{n}$, for all $n \geq 1$. Fix $n \geq 1$. By self-similarity, for any Borel set $E$,

$$
\nu_{\lambda}(E)=\sum_{x \in \mathcal{A}_{\lambda}^{(n)}} \nu_{\lambda}\left(\frac{E-x}{\lambda^{n}}\right) \cdot 2^{-n} .
$$

Thus, the measure $\nu_{\lambda}$ is a sum of $2^{n}$ "pieces," each having the measure $2^{-n}$. These pieces are supported on $\lambda^{n} A_{\lambda}+x$, for $x \in \mathcal{A}_{\lambda}^{(n)}$. Note that the supports of the pieces lie in $B_{C_{1}|\lambda|^{n}}(x)$. It follows that for any $z \in \mathbb{C}$ we have $\nu_{\lambda}\left(B_{C_{1}|\lambda|^{n}}(z)\right) \leq N \cdot 2^{-n}$, where $N$ is the number of points in $\mathcal{A}_{\lambda}^{(n)}$ that lie in $B_{2 C_{1}|\lambda|^{n}}(z)$. Since the separation between those points is at least $c \cdot 2^{-n / 2}$, we have $N\left(c^{2} / 4\right) \cdot 2^{-n} \leq 4 C_{1}^{2}|\lambda|^{2 n}$, whence $\nu_{\lambda}\left(B_{r}(z)\right) \leq\left(16 / c^{2}\right) r^{2}$, as desired. 
Proof of (4.4). Suppose that there is a point in $\mathcal{A}_{\lambda}^{(n)}$ having two different representations. Then there is a non-trivial polynomial $p(z)=a_{0}+a_{1} z+$ $\cdots+a_{n-1} z^{n-1}$, with $a_{k} \in\{-1,0,1\}$, such that $p(\lambda)=0$. The polynomial $q(z)=z^{n-1} p\left(z^{-1}\right)$ vanishes at $\theta=\lambda^{-1}$, hence the minimal polynomial of $\theta$ divides $q$. This is a contradiction, since $q$ has all coefficients of modulus less than or equal to one and the minimal polynomial of $\theta$ has the constant term \pm 2 .

Proof of (4.5). Let $x, y \in \mathcal{A}_{\lambda}^{(n)}, x \neq y$. We have

$$
\frac{1}{2}(x-y)=a_{0}+a_{1} \lambda+\cdots+a_{n-1} \lambda^{n-1}=: p(\lambda),
$$

where $a_{i} \in\{-1,0,1\}$ are not all zeros. Let $q(z)=z^{n-1} p\left(z^{-1}\right)$. Let $\theta_{1}=\theta$, $\theta_{2}=\bar{\theta}$, and let $\theta_{3}, \ldots, \theta_{m}$ be the remaining algebraic conjugates of $\theta$. The product $\prod_{j=1}^{m} q\left(\theta_{j}\right)$ is an integer, since it is a value of a symmetric polynomial on the roots of the minimal polynomial of $\theta$. We know that $q(\theta) \neq 0$ by the proof of (4.4), so $q\left(\theta_{j}\right) \neq 0$ for all $j \leq m$, since $q$ has integer coefficients.

Thus,

$$
\left|\prod_{j=1}^{m} q\left(\theta_{j}\right)\right| \geq 1 .
$$

Since $\left|\theta_{j}\right|>1$ by assumption, we have

$$
\left|q\left(\theta_{j}\right)\right| \leq\left|\theta_{j}\right|^{n-1}+\left|\theta_{j}\right|^{n-2}+\cdots<\frac{\left|\theta_{j}\right|^{n}}{\left|\theta_{j}\right|-1} .
$$

The constant term of the minimal polynomial is \pm 2 , so

$$
\left|\prod_{j=3}^{m} \theta_{j}\right|=2|\theta|^{-2} .
$$

In view of (4.8) and (4.9),

$$
\begin{aligned}
|q(\theta)|^{2} & =q(\theta) q(\bar{\theta}) \geq \prod_{j=3}^{m}\left|q\left(\theta_{j}\right)\right|^{-1} \\
& \geq \prod_{j=3}^{m}\left(\left|\theta_{j}\right|-1\right)\left|\theta_{j}\right|^{-n} \\
& =2^{-n}|\theta|^{2 n} \prod_{j=3}^{m}\left(\left|\theta_{j}\right|-1\right) .
\end{aligned}
$$

Now (4.7) implies

$$
|x-y|=2|p(\lambda)|=2|\theta|^{-n+1}|q(\theta)| \geq c \cdot 2^{-n / 2},
$$


as desired, where $c=2|\theta|\left(\prod_{j=3}^{m}\left(\left|\theta_{j}\right|-1\right)^{1 / 2}\right.$.

Proof of Proposition 2.0(i). This statement is immediate from the following lemma. Recall that $R_{a, b}$ is the rectangle with the vertices $\pm a \pm i b$.

Lemma 4.1. For any $\lambda \in \Omega$ there exist $a>1, b>2^{-1 / 2}$ such that $R_{a, b} \subset$ $\left(\lambda R_{a, b}-1\right) \cup\left(\lambda R_{a, b}+1\right)$.

The proof of this lemma is analogous to that of Lemma 3.3, so we omit it.

Proof of Proposition 2.6 (ii) is the same as in [14, p.424]; we include it for completeness. We prove the first statement; the second one is proved similarly. Let $\lambda=r e^{\pi i \frac{m}{n}}$, with $G C D(m, n)=1$. By (2.1),

$$
A_{\lambda}=A_{\lambda^{n}}+\lambda A_{\lambda^{n}}+\cdots+\lambda^{n-1} A_{\lambda^{n}} .
$$

Note that $\lambda^{n}= \pm r^{n}$ and $A_{z}=A_{-z}$, so $A_{\lambda^{n}}=A_{r^{n}} \subset \mathbb{R}$. Moreover, for $r \in\left[2^{-1 / n}, 1\right)$ we have $r^{n} \geq \frac{1}{2}$, so $A_{r^{n}}$ is a line segment of length $a=\frac{2}{1-r^{n}}$. Now we see that (4.10) represents a Minkowski sum of $n$ line segments making the angles $\frac{\pi k}{n}, k=0, \ldots, n-1$, with the horizontal. This implies the desired statement.

\section{RESUlts FOR A TYPICAL $\lambda$}

On the proof of Proposition 2.7. In [3, Thm 2] it is proved that if a power series $1+\sum_{n=1}^{\infty} a_{n} z^{n}$, with $a_{n} \in[-1,1]$, has $k$ roots (counting with multiplicities) in a disc of radius $r$, then

$$
r \geq k^{-1 / 2 k}\left(1+\frac{1}{k}\right)^{-\frac{1}{2}(1+1 / k)} .
$$

The presence of a non-real double root $\lambda$ implies that there are at least four roots in the disc of radius $|\lambda|+\varepsilon$, since the power series has real coefficients and $\bar{\lambda}$ has to be a double root as well. Substituting $k=4$ into the formula yields the desired estimate.

Remark. The proof of (5.1) is remarkably simple; it is based on Jensen's Formula, concavity of the logarithm, and Parseval's Formula. The bound $2 \cdot 5^{-5 / 8} \approx .73143$ obtained this way is quite good: Pinner 23 gave an example of a power series with coefficients in $[-1,1]$ having a double zero of modulus $\approx .75361$.

Using Proposition 2.7, all the theorems from Subsection 2.1.4 follow by standard methods. We only indicate how to deduce Theorem 2.10(ii). 
By (4.1), for any $k \geq 2$,

$$
\widehat{\nu}_{\lambda}(\xi)=\widehat{\nu}_{\lambda^{k}}(\xi) \widehat{\nu}_{\lambda^{k}}(\lambda \xi) \cdot \ldots \cdot \widehat{\nu}_{\lambda^{k}}\left(\lambda^{k-1} \xi\right) .
$$

Suppose that $\nu_{\lambda^{k}}$ has a density in $L^{2}\left(\mathbb{R}^{2}\right)$. Then $\widehat{\nu}_{\lambda^{k}} \in L^{2}\left(\mathbb{R}^{2}\right)$ by Plancherel's Theorem, hence $\widehat{\nu}_{\lambda} \in L^{2 / k}\left(\mathbb{R}^{2}\right)$ by (5.2). Since $\nu_{\lambda}$ is a probability measure, $\widehat{\nu}_{\lambda}$ is also bounded, so $\widehat{\nu}_{\lambda} \in L^{1}\left(\mathbb{R}^{2}\right)$. Applying the Inverse Fourier Transform, we conclude that $\nu_{\lambda}$ is absolutely continuous with a continuous density. Thus, for any $k \geq 2$,

$$
\frac{d \nu_{\lambda^{k}}}{d x} \in L^{2}\left(\mathbb{R}^{2}\right) \Rightarrow \frac{d \nu_{\lambda}}{d x} \in C\left(\mathbb{R}^{2}\right) .
$$

Now Theorem 2.10(i) implies Theorem 2.10(ii).

Acknowledgement. We are indebted to Christoph Bandt for motivating questions, many useful discussions, and help with the figures. Thanks also to David Boyd and David Garth for valuable information concerning complex Pisot numbers.

\section{REFERENCES}

1. C. Bandt, On the Mandelbrot set for pairs of linear maps, Nonlinearity 15 (2002), $1127-1147$.

2. M. F. Barnsley, Fractals Everywhere, Academic Press, 1988.

3. F. Beaucoup, P. Borwein, D. W. Boyd, and C. Pinner, Multiple roots of $[-1,1]$ power series, J. London Math. Soc.(2) 57 (1998), 135-147.

4. M. F. Barnsley and A. N. Harrington, A Mandelbrot set for pairs of linear maps, Phisica 15D (1985), 421-432.

5. T. Bousch, Connexité locale et par chemins hölderiens pour les systèmes itérés de fonctions, Preprint, 1993.

6. M. J. Bertin, A. Decomps-Guilloux, M. Grandet-Hugot, M. Pathiaux-Delefosse, J. P. Shreiber, Pisot and Salem numbers. Birkhäuser (1992).

7. C. Chamfy, Fonctions méromorphes dans le cercle-unité et leurs séries de Taylor, Ann. Inst. Fourier 8 (1958), 211-251.

8. P. Erdős, On a family of symmetric Bernoulli convolutions, Amer. J. Math. 61 (1939), 974-975.

9. K. J. Falconer, Dimensions and measures of quasi self-similar sets, Proc. Amer. Math. Soc. 106 (1989), 543-554.

10. K. J. Falconer, Fractal Geometry: Mathematical Foundations and Applications, Wiley, 1990.

11. A. M. Garsia, Arithmetic properties of Bernoulli convolutions, Trans. Amer. Math. Soc. 102 (1962), 409-432.

12. D. Garth, Complex Pisot numbers of small modulus, Preprint, 2002.

13. J. E. Hutchinson, Fractals and self-similarity, Indiana Univ. Math. J. 30 (1981), 713747. 
14. K.-H. Indlekofer, A. Járai, and I. Kátai, On some properties of attractors generated by iterated function systems, Acta Sci. Math. (Szeged) 60 (1995), 411-427.

15. K.-H. Indlekofer, I. Kátai, and P. Racsko, Some remarks on generalized number systems, Acta Sci. Math. (Szeged) $\mathbf{5 7}$ (1993), 543-553.

16. L. Jonker and J. J. P. Veerman, Semi-continuity of dimension and measure of locally scaling fractals, Fundamenta Mat., to appear.

17. A. M. Odlyzko and B. Poonen, Zeros of polynomials with 0,1 coefficients, L'Enseignement Math. 39 (1993), 317-348.

18. Y. Peres, W. Schlag, Smoothness of projections, Bernoulli convolutions, and the dimension of exceptions, Duke Math. J. 102 (2000), 193-251.

19. Y. Peres, W. Schlag, B. Solomyak, Sixty years of Bernoulli convolutions, Fractal Geometry and Stochastics II, C. Bandt, S. Graf, and M. Zähle (editors), Progress in Probability Vol. 46, 39-65, Birkhäuser, 2000.

20. Y. Peres, K. Simon and B. Solomyak, Self-similar sets of zero Hausdorff measure and positive packing measure, Israel J. Math. 117 (2000), 353-379.

21. Y. Peres, B. Solomyak, Absolute continuity of Bernoulli convolutions, a simple proof, Math. Research Letters 3, no. 2 (1996), 231-239.

22. Y. Peres, B. Solomyak, Self-similar measures and intersections of Cantor sets, Trans. Amer. Math. Soc. 350, no. 10 (1998), 4065-4087.

23. C. Pinner, Double roots of $[-1,1]$ power series and related matters, Math. Comp. 68, no. 227 (1999), 1149-1178.

24. M. Pollicott, K. Simon, The Hausdorff dimension of $\lambda$-expansions with deleted digits, Trans. Amer. Math. Soc. 347, no. 3 (1995), 967-983.

25. A. Schief, Separation properties for self-similar sets. Proc. Amer. Math. Soc. 122 (1994), 111-115.

26. C. L. Siegel, Algebraic integers whose conjugates lie in the unit circle, Duke Math. J. 11 (1944), 597-602.

27. K. Simon and B. Solomyak, On the dimension of self-similar sets, Fractals 10 (2002), 59-65.

28. B. Solomyak, On the random series $\sum \pm \lambda^{i}$ (an Erdös problem), Annals of Math. 142 (1995), 611-625.

29. B. Solomyak, Measure and dimension of some fractal families, Math. Proc. Cambridge Phil. Soc. 124, no. 3 (1998), 531-546.

Department of Mathematics, University of Washington, Seattle, WA 981954350

solomyak@math . washington . edu

hxu@math. washington.edu 